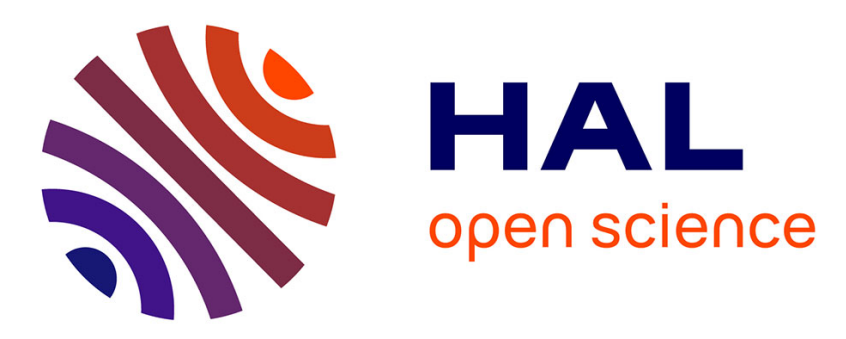

\title{
Interconnection through u-average passivity in discrete time
}

\author{
Alessio Moreschini, Mattia Mattioni, Salvatore Monaco, Dorothée \\ Normand-Cyrot
}

\section{- To cite this version:}

Alessio Moreschini, Mattia Mattioni, Salvatore Monaco, Dorothée Normand-Cyrot. Interconnection through u-average passivity in discrete time. CDC 2019 - 58th IEEE Conference on Decision and Control, IEEE, Dec 2019, Nice, France. 10.1109/CDC40024.2019.9029357 . hal-02350440

\author{
HAL Id: hal-02350440 \\ https://hal.science/hal-02350440
}

Submitted on 6 Nov 2019

HAL is a multi-disciplinary open access archive for the deposit and dissemination of scientific research documents, whether they are published or not. The documents may come from teaching and research institutions in France or abroad, or from public or private research centers.
L'archive ouverte pluridisciplinaire HAL, est destinée au dépôt et à la diffusion de documents scientifiques de niveau recherche, publiés ou non, émanant des établissements d'enseignement et de recherche français ou étrangers, des laboratoires publics ou privés. 


\title{
Interconnection through $u$-average passivity in discrete time
}

\author{
Alessio Moreschini ${ }^{1,2}$, Mattia Mattioni ${ }^{1}$, Salvatore Monaco $^{1}$ and Dorothée Normand-Cyrot ${ }^{2}$
}

\begin{abstract}
The paper discusses the preservation of $u$-average passivity throughout suitable interconnection. The concept of power preserving connection is introduced. It is instrumental to ensure $u$-average passivity of the interconnected system with respect to new external controls.
\end{abstract}

Index Terms-Nonlinear output feedback, Algebraic/geometric methods, Sampled-data control.

\section{INTRODUCTION}

The notions of dissipativity or passivity emerged in the analysis of physical systems to formalize the energy behavior induced by dissipating components [1], [2].

From the very first pioneering works on these topics by Willems, this concept has been proved to be fundamental for the analysis and control of nonlinear systems at large also because of the intrinsic connection between energy dissipation and Lyapunov-stability theory [3]. An important feature characterizing passivity is that when suitably interconnecting passive systems the overall system is still passive. This inspired a huge number of control techniques typically exploited in control engineering [2], [4]. Indeed, these interconnecting properties lead to nowadays well consolidated methodologies making reference to the so-called Passivity-Based Control (PBC); among many, the role and the properties of the interconnection are extensively exploited in Interconnection and Damping Assignment (IDA-PBC) and Control by Interconnection (CbI) [5]-[7]. Moreover, a variety of problems involving complex systems can be recast in the framework of energy-dissipation by modeling the complexity as a suitable power preserving interconnection; as an example, multi-agent and networked systems can be seen as interconnected passive subsystems [8]-[11].

All of this holds for continuous-time systems for which a consolidated body of methodologies for control and design has been proposed throughout the last decades. On the other hand, in the digitalization era discrete-time systems are becoming more and more demanded to model the information world. Accordingly, passivity and dissipation analysis are fundamental whereas several problems are still unsolved due to notable pathological issues arising in discrete time. In this

Supported by Università degli Studi di Roma La Sapienza (Progetti di Ateneo 2018-Piccoli progetti RP11816436325B63) and by Université Franco-Italienne/Università Italo-Francese (Vinci Grants 2018 and 2019).

${ }^{1}$ Dipartimento di Ingegneria Informatica, Automatica e Gestionale A. Ruberti (La Sapienza University of Rome); Via Ariosto 25, 00185 Rome, Italy \{alessio.moreschini, mattia.mattioni, salvatore.monaco\}euniromal.it.

${ }^{2}$ Laboratoire de Signaux et Systèmes (L2S, CNRS); 3, Rue Joliot Curie, 91192, Gif-sur-Yvette, France \{alessio.moreschini, dorothée. normand-cyrot \}al2s.centralesupelec.fr respect, a different concept of passivity has been introduced in [12] and referred to as average passivity; this notion involves passivity with respect to a new output defined as the average of the actual output over the control effort. This new discrete-time passivity concept solves part of the fundamental issues related to dissipative discrete-time systems as the one related to the necessity of a nonzero input-output link [13] [15].

In the framework of discrete-time $u$-average passive systems, a complete understanding of the properties under elementary interconnections is still unclear. The purpose of this paper is hence to make a first step in this direction by investigating the dissipating properties of the interconnection of average passive discrete-time systems through the corresponding $u$-average outputs. In doing so, the properties of the interconnection preserving average passivity of the overall systems are discussed with special emphasis toward power preserving patterns between the inputs and the $u$ average outputs rendering the exchange of energy lossless. By exploiting $u$-average passivity thus we characterize the family of power preserving patterns. The standard cases of parallel and feedback interconnection are investigated. In the latter case we show that, for $u$-average passivity to be preserved by the interconnected system, a particular power preserving pattern needs to be detailed. The results are specified to the LTI case for a deeper understanding of the involved computation and illustrated through the case of interconnected discrete-time van der Pol-like oscillators.

The paper is organized as follows. Preliminaries on discrete dynamics and average passivity are given in Section II. Parallel and feedback interconnections of nonlinear discretetime systems are investigated in Section III. An academic example is discussed in Section IV to illustrate the average passivity properties under feedback interconnection of $u$ average passive systems. Section V concludes the paper with future perspectives.

\section{Preliminaries}

$\mathbb{R}$ and $\mathbb{N}$ denote, respectively, the set of real and natural numbers including 0 . For all vectors $v \in \mathbb{R}^{n},|v|$ and $v^{\top}$ define the norm and transpose of $v$ respectively. Given $v \in \mathbb{R}^{n}$, the square norm is defined as $\|v\|^{2}:=v^{\top} v$. $I_{d}$ and $I$ denote respectively the identity operator and the identity matrix. Given a smooth vector field over $\mathbb{R}^{n}$, the Lie derivative operator is defined as $\mathrm{L}_{f}=\sum_{i=1}^{n} f_{i}(x) \frac{\partial}{\partial x_{i}}$. Given two smooth vector fields $f, g$, one defines the Lie bracket as $[f, g]=\mathrm{L}_{f} \mathrm{~L}_{g}-\mathrm{L}_{g} \mathrm{~L}_{f}$. The arguments of the functions are dropped when clear from the context so that $x:=x(k)$ with $k \geq 0$. 


\section{A. Differential/Difference Representation}

As discussed in [16], the following couple of differentialdifference equations describes a nonlinear discrete-time single-input dynamics $\Sigma_{d}$

$$
\begin{aligned}
x^{+} & =F_{0}(x) \\
\frac{\partial x^{+}(u)}{\partial u} & =G\left(x^{+}(u), u\right) \quad \text { with } \quad x^{+}(0)=x^{+}
\end{aligned}
$$

where $F_{0}(\cdot)$ is a $\mathbb{R}^{n}$-valued smooth map and $G(\cdot, u)$ is a vector field on $\mathbb{R}^{n}$, parameterized by $u \in U \subset \mathbb{R}$ and assumed complete. When the initial condition $x^{+}(0)$ is fixed, completeness of $G(\cdot, u)$ ensures integrability of (1b) so recovering the usual representation in the form of a map. In fact, for any pair $(x, u) \in \mathbb{R}^{n} \times \mathbb{R}$, denoting by $x^{+}(u)$ any curve in $\mathbb{R}^{n}$ parameterized by $u \in \mathbb{R}$, one gets

$$
x^{+}(u)=F(x, u)=x^{+}(0)+\int_{0}^{u} G\left(x^{+}(w), w\right) \mathrm{d} w
$$

with initial condition $x^{+}(0)=F_{0}(x)$. Accordingly, setting $x=x(k)$ and $u=u(k)$, one gets $x(k+1)=x^{+}(u(k))$ for all times $k \geq 0$. It is a matter of computation to verify that a given smooth map $F(x, u)$ can be described by equations of the form (1) provided $F(\cdot, 0)$ is invertible.

In the following, we assume that $\Sigma_{d}$ with input $u=0$ possesses an equilibrium at $x=0\left(F_{0}(0)=0\right)$. Some manipulations over the trajectories associated with dynamics (1) are instrumental. At first, by expanding $G(\cdot, u)$ in powers of $u$ as $G(\cdot, u)=G_{1}(\cdot)+\sum_{i \geq 1} \frac{u^{i}}{i !} G_{i+1}(\cdot)$ one gets a family of control vector fields over $\mathbb{R}^{n}$, the $G_{i}^{\prime} s$, characterizing the geometric structure of the flow associated to the solution of the differential equation (1b) (see [16] for further details). In this respect, one gets that (2) rewrites for all $(x, u) \in \mathbb{R}^{n} \times \mathbb{R}$ as

$$
x^{+}(u)=\left.e^{u \mathcal{G}(\cdot, u)} I d\right|_{x^{+}}
$$

where $e^{u \mathcal{G}(\cdot, u)}$ is the flow associated with $G(\cdot, u)$ which is characterized by the exponent series $u \mathcal{G}(\cdot, u)$ defined as

$$
u \mathcal{G}(\cdot, u)=u G_{1}+\frac{u^{2}}{2} G_{2}+\frac{u^{3}}{3 !}\left(G_{3}+\frac{1}{2}\left[G_{1}, G_{2}\right]\right)+O\left(u^{4}\right) .
$$

As usual, the notation $O\left(u^{p}\right)$ for any integer $p \geq 1$, defines the remaining higher order terms in the series expansion in $u$. From (3), one computes the first terms in the expansion of $F(\cdot, u)$ in $u$ as follows

$$
\begin{aligned}
& F(x, u)=\left.e^{u G_{1}+\frac{u^{2}}{2} G_{2}+\frac{u^{3}}{3 !}\left(G_{3}+\frac{1}{2}\left[G_{1}, G_{2}\right]\right)+O\left(u^{4}\right)} I_{d}\right|_{F_{0}(x)}= \\
& F_{0}(x)+\left.u \mathrm{~L}_{G_{1}} I_{d}\right|_{F_{0}(x)}+\left.\frac{u^{2}}{2}\left(\mathrm{~L}_{G_{1}}^{2}+\mathrm{L}_{G_{2}}\right) I_{d}\right|_{F_{0}(x)}+O\left(u^{3}\right) .
\end{aligned}
$$

As a consequence of the form (1), given any smooth enough map $h(\cdot): \mathbb{R}^{n} \rightarrow \mathbb{R}$, one gets

$$
h\left(x^{+}(u)\right)=h\left(F_{0}(x)\right)+\int_{0}^{u} \mathrm{~L}_{G(\cdot, w)} h\left(x^{+}(w)\right) \mathrm{d} w
$$

with $\mathrm{L}_{G(\cdot, w)} h\left(x^{+}(w)\right)=\mathrm{L}_{G_{1}} h\left(F_{0}(x)\right)+w\left(\mathrm{~L}_{G_{1}}^{2}+\right.$ $\left.\mathrm{L}_{G_{2}}\right) h\left(F_{0}(x)\right)+O\left(w^{2}\right)$. In a more compact form, exploiting the flow associated with the solution to the differential equation (1b), one gets

$$
\begin{aligned}
h\left(x^{+}(u)\right)= & \left.e^{u \mathcal{G}(\cdot, u)} h\right|_{x^{+}}=h\left(F_{0}(x)\right)+u \mathrm{~L}_{G_{1}} h\left(F_{0}(x)\right) \\
& +\frac{u^{2}}{2}\left(\mathrm{~L}_{G_{1}}^{2} h\left(F_{0}(x)\right)+\mathrm{L}_{G_{2}} h\left(F_{0}(x)\right)\right)+O\left(u^{3}\right) .
\end{aligned}
$$

\section{B. Average passivity in discrete time}

The concept of average passivity has been introduced in [12] to relax the necessity of a direct input/output link, that is unavoidable in discrete time when referring to a more classical passivity concept [17]. The following definition is recalled denoting by $\Sigma_{d}(h)$ the discrete-time system (1) with output map $h(\cdot): \mathbb{R}^{n} \rightarrow \mathbb{R}$.

Definition 2.1 (u-average passivity): Given $\Sigma_{d}(h)$, let the $u$-average output mapping be defined as

$$
h^{a v}(x, u):=\frac{1}{u} \int_{0}^{u} h\left(x^{+}(w)\right) \mathrm{d} w
$$

for any $(x, u) \in \mathbb{R}^{n} \times \mathbb{R}$, with $h^{a v}(x, 0):=h\left(x^{+}(0)\right)$ and $x^{+}(0)=F_{0}(x) . \Sigma_{d}(h)$ is said to be u-average passive if there exists a positive semi-definite function $S(\cdot): \mathbb{R}^{n} \rightarrow$ $\mathbb{R}_{\geq 0}$ (the storage function) such that, for all $(x, u) \in \mathbb{R}^{n} \times \mathbb{R}$

$$
S\left(x^{+}(u)\right)-S(x) \leq \int_{0}^{u} h\left(x^{+}(w)\right) \mathrm{d} w=u h^{a v}(x, u) .
$$

It is worth mentioning that $u$-average passivity of (1) with respect to $h(\cdot)$ is equivalent to usual passivity of (1) with respect to $h^{a v}(\cdot, u)$. As discussed in [12], a necessary condition for $u$-average passivity is for $\Sigma_{d}(h)$ to possess relative degree $r_{d}=1$ (that is $\frac{\partial h\left(x^{+}(u)\right)}{\partial u} \neq 0$ ) and, thus, for $\Sigma_{d}\left(h^{a v}(\cdot, u)\right)$ to possess relative degree $r_{d}^{a v}=0$ (i.e., $\frac{\partial h^{a v}(\cdot, u)}{\partial u} \neq 0$ ) at least in a neighborhood of the equilibrium.

In [18], [19], the notion of $u$-average passivity from some nominal control value $\bar{u}$ has been introduced to deal with incremental-like passivity properties in discrete time. The following definition is hence recalled.

Definition 2.2 (u-average passivity from $\bar{u}): \Sigma_{d}(h)$ is $u$ average passive from $\bar{u} \in \mathbb{R}$ if there exists a positive semidefinite function $S(\cdot): \mathbb{R}^{n} \rightarrow \mathbb{R}_{\geq 0}$ (the storage function, with $S(0)=0)$ such that, for all $(x, u) \in \mathbb{R}^{n} \times \mathbb{R}$,

$$
S\left(x^{+}(u)\right)-S(x) \leq(u-\bar{u}) h_{\bar{u}}^{a v}(x, u) .
$$

with $h_{\bar{u}}^{a v}(x, u)=\frac{1}{u-\bar{u}} \int_{\bar{u}}^{u} h\left(x^{+}(w)\right) \mathrm{d} w$.

Remark 2.1: From (7), it is clear that, when $\bar{u} \equiv 0, u$ average passivity from $\bar{u}$ recovers classical $u$-average passivity.

\section{INTERCONNECTION OF PASSIVE SYSTEMS}

For $i=1,2$, consider the respective discrete-time systems $\Sigma_{i}\left(h_{i}\right)$ with state dynamics

$$
x_{i}^{+}\left(u_{i}\right)=F_{i}\left(x_{i}, u_{i}\right)=F_{i}\left(x_{i}, 0\right)+\int_{0}^{u_{i}} G_{i}\left(x_{i}^{+}(w), w\right) \mathrm{d} w
$$


and output maps $h_{i}(\cdot): \mathbb{R}^{n} \rightarrow \mathbb{R}$; assume $u$-average passivity with respective averaged outputs $h_{i}^{a v}\left(x_{i}, u_{i}\right)=$ $\int_{0}^{u_{i}} h_{i}\left(x_{i}(w)\right) \mathrm{d} w$ and storage functions $S_{i}(\cdot): \mathbb{R}^{n} \rightarrow \mathbb{R}_{\geq 0}$. For the sake of compactness, we denote $x=\operatorname{col}\left(x_{1}, x_{2}\right)$.

In what follows, given two discrete-time dynamics of the form (8), we investigate the average passivity properties arising when an input/output connection is established through an interconnecting pattern between the inputs and the associated outputs. More precisely, we formalize the interconnection between two systems through an operator

$$
\Phi_{c}:\left(h_{1}\left(x_{1}\right), h_{2}\left(x_{2}\right), u_{1}, u_{2}\right) \mapsto\left(\begin{array}{l}
u_{1} \\
u_{2}
\end{array}\right)
$$

describing the interconnecting pattern that links the outputs $\left(h_{1}(\cdot), h_{2}(\cdot)\right)$ to the inputs $\left(u_{1}, u_{2}\right)$ via $u$-averaging.

At first, we define a power preserving pattern ensuring no loss of energy throughout the interconnection process (lossless connection). The following definition is instrumental.

Definition 3.1: The interconnection between $\Sigma_{1}\left(h_{1}\right)$ and $\Sigma_{2}\left(h_{2}\right)$ given in (8) for $i=1,2$ is said power preserving if there exists an interconnecting pattern (9) satisfying

$$
\int_{0}^{u_{1}} h_{1}\left(x_{1}^{+}(w)\right) d w+\int_{0}^{u_{2}} h_{2}\left(x_{2}^{+}(w)\right) d w=0
$$

when $\left(\begin{array}{l}u_{1} \\ u_{2}\end{array}\right)=\Phi_{c}\left(h_{1}\left(x_{1}\right), h_{2}\left(x_{2}\right), u_{1}, u_{2}\right)$.

By definition of $u$ average passivity, the integral form (10) rewrites as

$$
u_{1} h_{1}^{a v}\left(x_{1}, u_{1}\right)+u_{2} h_{2}^{a v}\left(x_{2}, u_{2}\right)=0
$$

so highlighting that the interconnection involves the $u$ average outputs. In fact, among these power preserving interconnections, the simplest way to solve (11) is to set

$$
\Phi_{c}\left(h_{1}\left(x_{1}\right), h_{2}\left(x_{2}\right), u_{1}, u_{2}\right)=\left(\begin{array}{cc}
0 & -1 \\
1 & 0
\end{array}\right)\left(\begin{array}{l}
h_{1}^{a v}\left(x_{1}, u_{1}\right) \\
h_{2}^{a v}\left(x_{2}, u_{2}\right)
\end{array}\right)
$$

so recovering the classical power preserving interconnection expressed with respect to average outputs. The solution to the implicit equality (11) defines a preliminary power preserving state-feedback that we denote $\bar{u}=\bar{u}(x)$ This underlines that the internal exchange of energy provided by the pattern $\Phi_{c}$ via $\bar{u}$ is preserved under the interconnection.

\section{A. Parallel interconnection of passive systems}

Let us study the average passivity properties of the parallel interconnection of (8) for $i=1,2$ that is when setting

$$
u=u_{1}=u_{2} \quad h(x)=h_{1}\left(x_{1}\right)+h_{2}\left(x_{2}\right) .
$$

Theorem 3.1: Consider the parallel interconnection of $u$ average passive systems (8) with $i=1,2$. Then, the resulting system

$$
\begin{aligned}
& x_{1}^{+}(u)=F_{1}\left(x_{1}, u\right) \\
& x_{2}^{+}(u)=F_{2}\left(x_{2}, u\right)
\end{aligned}
$$

with output $h(x):=h_{1}\left(x_{1}\right)+h_{2}\left(x_{2}\right)$ is $u$-average passive with storage function $S(x)=S_{1}\left(x_{1}\right)+S_{2}\left(x_{2}\right)$.

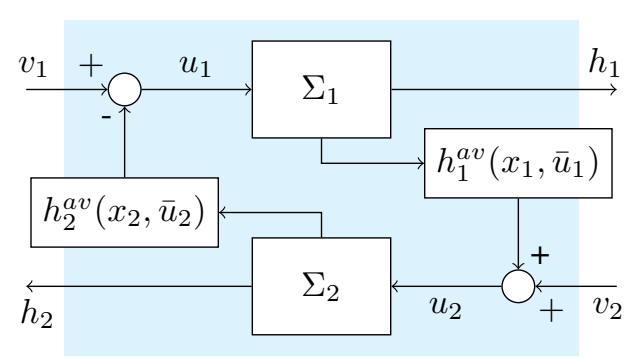

Fig. 1: Power preserving feedback interconnection between $\Sigma_{1}\left(h_{1}\right)$ and $\Sigma_{2}\left(h_{2}\right)$ via average outputs with external $v$.

Proof: Because each subsystem $\Sigma_{i}\left(h_{i}\right)$ in (8) $(i=1,2)$ $u$-average passive, one gets that

$$
\begin{aligned}
\Delta S(x):= & S\left(x^{+}(u)\right)-S(x) \\
& \leq \int_{0}^{u_{1}} h_{1}\left(x_{1}^{+}(w)\right) \mathrm{d} w+\int_{0}^{u_{2}} h_{2}\left(x_{2}^{+}(w)\right) \mathrm{d} w .
\end{aligned}
$$

Because $u=u_{1}=u_{2}$ one gets

$$
\begin{aligned}
\Delta S(x) & \leq \int_{0}^{u}\left[h_{1}\left(x_{1}^{+}(w)\right)+h_{2}\left(x_{2}^{+}(w)\right)\right] \mathrm{d} w \\
& =\int_{0}^{u} h\left(x^{+}(w)\right) \mathrm{d} w=u h^{a v}(x, u)
\end{aligned}
$$

and thus the result.

The above result shows that, when considering the parallel interconnection of $u$-average passive systems (8), passivity in the $u$-average sense is preserved with respect to the natural output induced by the sum of the single ones. It is worth to note that the averaged output, in this case, is also equal to the sum of the averaged outputs associated to (8) when setting $u=u_{i}$ for $i=1,2$.

Remark 3.1: We note that the parallel interconnection (13) is not power preserving in the sense of Definition 3.1.

\section{B. Feedback interconnection of passive systems}

Let us now define a feedback interconnection of (8) enhancing $u$-average passivity of the resulting system. To this end, let us consider the input $u=\operatorname{col}\left(u_{1}, u_{2}\right)$

$$
u=\Phi_{c}\left(h_{1}\left(x_{1}\right), h_{2}\left(x_{2}\right), \bar{u}\right)+v .
$$

with $v=\operatorname{col}\left(v_{1}, v_{2}\right)$ being external inputs and $\bar{u}=$ $\operatorname{col}\left(\bar{u}_{1}, \bar{u}_{2}\right)$ being the power preserving feedback computed as the solution to the interconnecting pattern

$$
\bar{u}=\Phi_{c}\left(h_{1}\left(x_{1}\right), h_{2}\left(x_{2}\right), \bar{u}\right)
$$

satisfying (10) (equivalently, (11)) in Definition 3.1. The following Lemma is instrumental.

Lemma 3.1: Consider the systems $\Sigma_{i}\left(h_{i}\right)$ being $u$-average passive with storage functions $S_{i}(\cdot): \mathbb{R}^{n} \rightarrow \mathbb{R}_{\geq 0}$. Consider the state power preserving interconnection (16). Then, the augmented system $\Sigma_{c}$

$$
\begin{aligned}
& x_{1}^{+}\left(u_{1}\right)=F_{1}\left(x_{1}, u_{1}\right) \\
& x_{2}^{+}\left(u_{2}\right)=F_{2}\left(x_{2}, u_{2}\right)
\end{aligned}
$$


with output

$$
h_{c}(x):=\operatorname{col}\left(h_{1}\left(x_{1}\right), h_{2}\left(x_{2}\right)\right)
$$

is $u$-average passive from $\bar{u}=\bar{u}(x)$, solution to (16), and storage function $S_{c}(x):=S_{1}(x)+S_{2}(x)$ verifying the dissipation inequality

$$
\Delta S_{c}(x) \leq(u-\bar{u})^{\top} h_{c, \bar{u}}^{a v}(x, u),
$$

with

$$
h_{c, \bar{u}}^{a v}(x, u)=\left(\begin{array}{l}
\frac{1}{u_{1}-\bar{u}_{1}} \int_{\bar{u}_{1}}^{u_{1}} h_{1}\left(x_{1}^{+}(w)\right) \mathrm{d} w \\
\frac{1}{u_{2}-\bar{u}_{2}} \int_{\bar{u}_{2}}^{u_{2}} h_{2}\left(x_{2}^{+}(w)\right) \mathrm{d} w
\end{array}\right) .
$$

Proof: Under the internal state power-preserving interconnection $\bar{u}=\Phi_{c}\left(h_{1}\left(x_{1}\right), h_{2}\left(x_{2}\right), \bar{u}\right)$ solution to (11), one has

$$
\sum_{i=1}^{2} \int_{0}^{\bar{u}_{i}} h_{i}\left(x_{i}^{+}(w)\right) \mathrm{d} w=0 .
$$

Accordingly, by computing $\Delta S_{c}(x)=\Delta S_{c}\left(x^{+}(u)\right)-S(x)$ and exploiting $u$-average passivity of (8), one gets from (10)

$$
\begin{aligned}
\Delta S_{c}(x) & \leq \sum_{i=1}^{2} \int_{0}^{u_{i}} h_{i}\left(x_{i}^{+}(w)\right) \mathrm{d} w \\
& =\underbrace{\sum_{i=1}^{2} \int_{0}^{\bar{u}_{i}} h_{i}\left(x_{i}^{+}(w)\right) \mathrm{d} w}_{=0}+\sum_{i=1}^{2} \int_{\bar{u}_{i}}^{u_{i}} h_{i}\left(x_{i}^{+}(w)\right) \mathrm{d} w
\end{aligned}
$$

and thus the results.

The following result can be thus proved.

Theorem 3.2: Let, for $i=1,2$, the systems $\Sigma_{i}\left(h_{i}\right)$ be $u$-average passive with respective storage functions $S_{i}(\cdot)$ : $\mathbb{R}^{n} \rightarrow \mathbb{R}_{\geq 0}$. Consider the input (15) with $\bar{u}=\bar{u}(x)$ being the power-preserving interconnection (16). Then, the interconnected system

$$
\begin{aligned}
& x_{1}^{+}\left(\bar{u}_{1}(x)+v_{1}\right)=F_{1}\left(x_{1}, \bar{u}_{1}(x)+v_{1}\right) \\
& x_{2}^{+}\left(\bar{u}_{2}(x)+v_{2}\right)=F_{2}\left(x_{2}, \bar{u}_{2}(x)+v_{2}\right)
\end{aligned}
$$

with output (18) is $u$-average passive with storage function $S_{c}(x):=S_{1}(x)+S_{2}(x)$. Namely, the dissipation inequality

$$
\Delta S(x) \leq v^{\top} h_{c, \bar{u}}(x, \bar{u}(x)+v)
$$

holds with average output

$$
h_{c, \bar{u}}(x, \bar{u}(x)+v)=\left(\begin{array}{l}
\frac{1}{v_{1}} \int_{0}^{v_{1}} h_{1}\left(x_{1}^{+}\left(\bar{u}_{1}+\ell\right)\right) \mathrm{d} \ell \\
\frac{1}{v_{2}} \int_{0}^{v_{2}} h_{2}\left(x_{2}^{+}\left(\bar{u}_{2}+\ell\right)\right) \mathrm{d} \ell
\end{array}\right) .
$$

Proof: By Lemma 3.1, u-average passivity of (20) from (16) holds. Thus, by plugging the input (15) into the dissipation inequality (19) one gets

$$
\begin{aligned}
\Delta S_{c}(x) & \leq \sum_{i=1}^{2} \int_{\bar{u}_{i}}^{\bar{u}_{i}+v_{i}} h_{i}\left(x_{i}^{+}(w)\right) \mathrm{d} w \\
& =\sum_{i=1}^{2} \int_{0}^{v_{i}} h_{i}\left(x_{i}^{+}\left(\bar{u}_{i}+\ell\right)\right) \mathrm{d} \ell=v^{\top} h_{c, \bar{u}}(x, \bar{u}(x)+v)
\end{aligned}
$$

so concluding the proof.
Remark 3.2: The input (15) is composed of two terms: the exogenous signal $v$ and an interconnecting feedback $\bar{u}=$ $\bar{u}(x)$ defining the pattern as the solution to (10). This latter term is a power preserving state feedback computed over the averaged outputs $h_{i}^{a v}\left(x_{i}, u_{i}\right)$ when setting the exogenous signal in (15) $v_{i}=0$ (that is $u=\bar{u}$ ). Such an interconnection is not realized through the direct input/averaged-output ports obtained through the feedback $u=u(x, v)$ solution to

$$
\left(\begin{array}{l}
u_{1} \\
u_{2}
\end{array}\right)=\left(\begin{array}{cc}
0 & -1 \\
1 & 0
\end{array}\right)\left(\begin{array}{l}
h_{1}^{a v}\left(x_{1}, u_{1}\right) \\
h_{2}^{a v}\left(x_{2}, u_{2}\right)
\end{array}\right)+v .
$$

Indeed, it is a matter of computations to verify that in that case, average passivity of the overall systems is not preserved by the interconnection whereas only classical passivity stands with respect to the output $H(x, v)=$ $\operatorname{col}\left(h_{1}^{a v}\left(x_{1}, u_{1}\right), h_{2}^{a v}\left(x_{2}, u_{2}\right)\right)$ with $\left(u_{1}, u_{2}\right)^{\top}$ solution to (23). Nevertheless, a direct input-output link is still needed for this.

Remark 3.3: Theorem 3.2 defines a family of feedback interconnections preserving $u$-average passivity of the overall system. As it is clear from (21), under an external source $v$ and for a fixed $\bar{u}$ in (16), the average outputs are defined starting from the same outputs of the single systems (8) but averaged over the new interconnected dynamics (20) deduced from (15). Thus, starting from average passivity of the single systems (8) with outputs $h_{i}\left(x_{i}\right)$, the interconnected system is $u$-average passive under the power preserving stated feedback $\bar{u}=\bar{u}(x)$ solving (16). A family of $u$ average outputs of the form (22) computed starting from $h_{i}\left(x_{i}\right)$ and associated to $\bar{u}=\bar{u}(x)$ exists with each element being parametrized by the choice of the interconnection patter $\Phi_{c}(\cdot)$ satisfying $(10)$.

Remark 3.4: When one of the systems involved in the interconnection (17) is not average passive, under certain conditions involving stability, one can prove that the corresponding interconnection through (15) provides the total energy variation

$\Delta S_{c}(x)=S_{c}\left(x^{+}(\bar{u})\right)-S_{c}(x)+\sum_{i=1}^{2} \int_{\bar{u}_{i}}^{\bar{u}_{i}+v_{i}} h_{i}\left(x_{i}^{+}(w)\right) \mathrm{d} w$

allowing to conclude that the interconnected system $\Sigma_{c}(h)$ is average passive if $S_{c}\left(x^{+}(\bar{u})\right) \leq S_{c}(x)$ yields for all $x \in \mathbb{R}^{2 n}$.

1) Computational aspects : The power preserving interconnecting feedback (16) is defined as the implicit solution of a nonlinear equality induced by the averaged outputs. Although exact computations are tough to be carried out in general, an approximate solution to (16) does exist (at least locally) as specified in the following proposition. To this end, assume, without loss of generality that for $i=1,2$, (8) possesses an equilibrium at the origin. For the sake of facility, let us assume the interconnecting pattern be defined by (12). Thus, the following result holds true.

Proposition 3.1: Consider, for $i=1,2$, the discrete-time systems $\Sigma_{i}\left(h_{i}\right)$ in (8) with equilibrium at $x=0$ and being $u$ average passive. Consider the interconnection (12). Then, for 
all $x$ in a neighborhood of the origin, the equality (16) with pattern (12) locally admits a solution $\bar{u}_{a}=\bar{u}_{a}(x)$ verifying $\bar{u}(0)=0$. Thus, the interconnected system (20) is locally passive when setting $u=\bar{u}_{a}(x)+v$.

Proof: First, consider the expansion of the average outputs associated to $h_{i}\left(x_{i}\right)$ as given, for $i=1,2$, as

$h_{i}^{a v}\left(x_{i}, u_{i}\right)=h_{i}\left(F_{i}\left(x_{i}, 0\right)\right)+u_{i} \mathrm{~L}_{G_{i}} h_{i}\left(F_{i}\left(x_{i}, 0\right)\right)+O\left(u_{i}^{2}\right)$,

where $O\left(u_{i}^{2}\right)$ contains all the remaining terms of a higher order of the control variable $u_{i}$. By substituting such an expansion in the equality (16) one gets

$$
\begin{aligned}
& \bar{u}_{1}=-h_{2}\left(F_{2}\left(x_{2}, 0\right)\right)+\bar{u}_{2} \mathrm{~L}_{G_{2}} h_{2}\left(F_{2}\left(x_{2}, 0\right)\right)+O\left(u_{2}^{2}\right) \\
& \bar{u}_{2}=h_{1}\left(F_{1}\left(x_{1}, 0\right)\right)+\bar{u}_{1} \mathrm{~L}_{G_{1}} h_{1}\left(F_{1}\left(x_{1}, 0\right)\right)+O\left(u_{1}^{2}\right)
\end{aligned}
$$

By invoking the implicit function theorem, for all $x$ in the neighborhood of the origin, an approximate solution exists because the non-singularity condition

$$
\left|\frac{\partial \Phi_{c}\left(h_{1}\left(x_{1}\right), h_{2}\left(x_{2}\right), u_{1}, u_{2}\right)}{\partial u}\right|_{u=0}=|A(x)| \neq 0
$$

with

$$
A(x):=\left(\begin{array}{cc}
1 & \mathrm{~L}_{G_{2}(\cdot, 0)} h_{2}\left(F_{2}\left(x_{2}, 0\right)\right) \\
-\mathrm{L}_{G_{1}(\cdot, 0)} h_{1}\left(F_{1}\left(x_{1}, 0\right)\right) & 1
\end{array}\right)
$$

holds true since each $\Sigma_{i}\left(h_{i}\right)$ in (8) has relative degree 1 . Accordingly, one gets local passivity from Theorem 3.2 when $u=\bar{u}_{a}(x)+v$ with

$$
\bar{u}_{a}(x)=A^{-1}(x)\left(\begin{array}{c}
-h_{2}\left(F_{2}\left(x_{2}, 0\right)\right) \\
h_{1}\left(F_{1}\left(x_{1}, 0\right)\right)
\end{array}\right) .
$$

2) The case of average passive LTI systems: Consider, for $i=1,2$, the case in which (8) are LTI that is

$$
\begin{aligned}
x_{i}^{+}\left(u_{i}\right) & =A_{i} x_{i}+B_{i} u_{i} \\
y_{i} & =C_{i} x_{i}
\end{aligned}
$$

with relative degree $r_{i}=1$, (i.e., $C_{i} B_{i} \neq 0$ ). Then, assuming (24) average passive with quadratic storage function $S_{i}(x)=$ $\frac{1}{2} x_{i}^{\top} P_{i} x_{i}$ and $P_{i}>0$, the average outputs get the form $h_{i}^{a v}\left(x_{i}, u_{i}\right)=C_{i} A_{i} x_{i}+\frac{1}{2} C_{i} B_{i} u_{i}$. In that case, the power preserving interconnection (12) is specified as

$$
\Phi_{c}(x)=\left(\begin{array}{cc}
\mathbf{0} & -C_{2} A_{2} \\
C_{1} A_{1} & \mathbf{0}
\end{array}\right) x+\left(\begin{array}{cc}
0 & -\frac{1}{2} C_{2} B_{2} \\
\frac{1}{2} C_{1} B_{1} & 0
\end{array}\right) u
$$

so that the equality (16) admits, because of the relative degree condition, a unique solution $\bar{u}=F x$ with

$$
\begin{aligned}
F & =\left(\begin{array}{ll}
F_{1} & F_{2} \\
F_{3} & F_{4}
\end{array}\right) \\
& =\left(\begin{array}{cc}
1 & \frac{1}{2} C_{2} B_{2} \\
-\frac{1}{2} C_{1} B_{1} & 1
\end{array}\right)^{-1}\left(\begin{array}{cc}
\mathbf{0} & -C_{2} A_{2} \\
C_{1} A_{1} & \mathbf{0}
\end{array}\right)
\end{aligned}
$$

When considering the pattern (12), one gets the overall system

$$
\begin{aligned}
x_{1}^{+}\left(\bar{u}_{1}+v_{1}\right) & =\left(A_{1}+B_{1} F_{1}\right) x_{1}+B_{1} F_{2} x_{2}+B_{1} v_{1} \\
x_{2}^{+}\left(\bar{u}_{2}+v_{2}\right) & =B_{2} F_{3} x_{1}+\left(A_{2}+B_{2} F_{4}\right) x_{2}+B_{2} v_{2} \\
y_{1} & =C_{1} x_{1}, \quad y_{2}=C_{2} x_{2}
\end{aligned}
$$

that is $u$-average passive with output provided as the average of (25c) along the interconnected system (25a)-(25b); namely, setting $S_{c}(x)=x_{1}^{\top} P_{1} x_{1}+x_{2}^{\top} P_{2} x_{2}$, one gets

$$
\Delta S_{c}(x) \leq v^{\top} h_{c, \bar{u}}^{a v}(x, \bar{u}(x)+v)
$$

with average output $\tilde{h}_{c, \bar{u}}^{a v}(x, v)=h_{c, \bar{u}}^{a v}(x, \bar{u}(x)+v)$,

$\tilde{h}_{c, \bar{u}}^{a v}(x, v)=\left(\begin{array}{l}C_{1}\left(A_{1}+B_{1} F_{1}\right) x_{1}+C_{1} B_{1} F_{2} x_{2}+\frac{1}{2} C_{1} B_{1} v_{1} \\ C_{2} B_{2} F_{3} x_{1}+C_{2}\left(A_{2}+B_{2} F_{4}\right) x_{2}+\frac{1}{2} C_{2} B_{2} v_{2}\end{array}\right)$.

Remark 3.5: When specifying the direct connection (23) to this case, that is under output-feedback connection, one gets $u_{c}=F x+D v$ with

$$
D=\left(\begin{array}{c}
D_{1} \\
D_{2}
\end{array}\right)=\left(\begin{array}{cc}
1 & \frac{1}{2} C_{2} B_{2} \\
-\frac{1}{2} C_{1} B_{1} & 1
\end{array}\right)^{-1},
$$

so yielding classical passivity of the overall system with

$$
H(x, v)=\left(\begin{array}{l}
C_{1}\left(A_{1}+\frac{1}{2} B_{1} F_{1}\right) x_{1}+\frac{1}{2} C_{1} B_{1} F_{2} x_{2}+\frac{1}{2} C_{1} B_{1} D_{1} v \\
\frac{1}{2} C_{2} B_{2} F_{3} x_{1}+C_{2}\left(A_{2}+\frac{1}{2} B_{2} F_{4}\right) x_{2}+\frac{1}{2} C_{2} B_{2} D_{2} v
\end{array}\right)
$$

which is not the average of the outputs $(25 \mathrm{c})$ deduced from the original systems under the resulting interconnection.

\section{ILlustratiVE EXAMPLE}

Let assume the interconnection between two nonlinear van der Pol oscillators in discrete time described by [20], [21],

$$
x_{i}^{+}(u)=x_{i}+\frac{1}{2}\left(J-R_{i}\left(x_{i}\right)\right)\left(x_{i}^{+}+x_{i}\right)+g_{i}\left(x_{i}\right) u_{i}
$$

with $x_{i}=\operatorname{col}\left(x_{i}^{1}, x_{i}^{2}\right) \in \mathbb{R}^{2}$ and, for $\mu_{i}>0$

$$
J=\left(\begin{array}{cc}
0 & 1 \\
-1 & 0
\end{array}\right), R\left(x_{1}\right)=\left(\begin{array}{cc}
0 & 0 \\
0 & -\mu_{i}\left(1-\left(x_{i}^{1}\right)^{2}\right)
\end{array}\right), B=\left(\begin{array}{l}
0 \\
1
\end{array}\right)
$$

$x_{i}^{+}=\left(I-A_{i}\left(x_{i}\right)\right)^{-1}\left(I+A_{i}\left(x_{i}\right)\right) x_{i}, g_{i}\left(x_{i}\right)=(I-$ $\left.A_{i}\left(x_{i}\right)\right)^{-1} B$ and $A_{i}\left(x_{i}\right)=\frac{1}{2}\left(J-R\left(x_{i}\right)\right)$. (26) is $u$-average passive with $u$-average output $h_{i}^{a v}\left(x_{i}, u_{i}\right)=g_{i}^{\top}\left(x_{i}\right)\left(x_{i}^{+}+\right.$ $\left.\frac{1}{2} g_{i}\left(x_{i}\right) u_{i}\right)$ and storage function $S_{i}\left(x_{i}\right)=\frac{1}{2} x_{i}^{\top} x_{i}$.

The two oscillators are feedback connected through (16) being exactly computed as

$$
\bar{u}=\frac{\left(\begin{array}{cc}
-\frac{1}{2}\left\|g_{2}\left(x_{2}\right)\right\|^{2} g_{1}^{\top}\left(x_{1}\right) & -g_{2}^{\top}\left(x_{2}\right) \\
g_{1}^{\top}\left(x_{1}\right) & -\frac{1}{2}\left\|g_{1}\left(x_{1}\right)\right\|^{2} g_{2}^{\top}\left(x_{2}\right)
\end{array}\right)}{1+\frac{1}{4}\left\|g_{1}\left(x_{1}\right)\right\|^{2}\left\|g_{2}\left(x_{2}\right)\right\|^{2}} x^{+}
$$

with $x^{+}=\operatorname{col}\left(x_{1}^{+}, x_{2}^{+}\right)$. Thus, in accordance with Theorem 3.2 , one gets that the overall interconnected system is average passive with average output $\tilde{h}_{c, \bar{u}}^{a v}(x, v)=h_{c, \bar{u}}^{a v}(x, \bar{u}(x)+v)$

$$
\tilde{h}_{c, \bar{u}}^{a v}(x, v)=\left(\begin{array}{l}
g_{1}^{\top}\left(x_{1}\right)\left(x_{1}^{+}+g_{1}\left(x_{1}\right) \bar{u}_{1}(x)\right)+\frac{1}{2}\left\|g_{1}\left(x_{1}\right)\right\|^{2} v_{1} \\
g_{2}^{\top}\left(x_{2}\right)\left(x_{2}^{+}+g_{2}\left(x_{2}\right) \bar{u}_{2}(x)\right)+\frac{1}{2}\left\|g_{2}\left(x_{2}\right)\right\|^{2} v_{2}
\end{array}\right) .
$$

Accordingly, under zero-state detectability, one can stabilize the system at the origin by introducing the damping feedback $v=-\kappa h_{c, \bar{u}}^{a v}(x, \bar{u}(x)+v)$ so that $v=v(x)$ is given by

$$
v=\kappa\left(\begin{array}{l}
\frac{g_{1}^{\top}\left(x_{1}\right)\left(x_{1}^{+}+g_{1}\left(x_{1}\right) \bar{u}_{1}(x)\right)}{1+\frac{\kappa}{2}\left\|g_{1}\left(x_{1}\right)\right\|^{2}} \\
\frac{g_{2}^{\top}\left(x_{2}\right)\left(x_{2}^{+}+g_{2}\left(x_{2}\right) \bar{u}_{2}(x)\right)}{1+\frac{\kappa}{2}\left\|g_{2}\left(x_{2}\right)\right\|^{2}}
\end{array}\right), \quad \kappa>0 .
$$

Simulations: For completeness, simulations of the discussed example are reported in Figure 2 by setting $\mu_{1}=0.8$, 

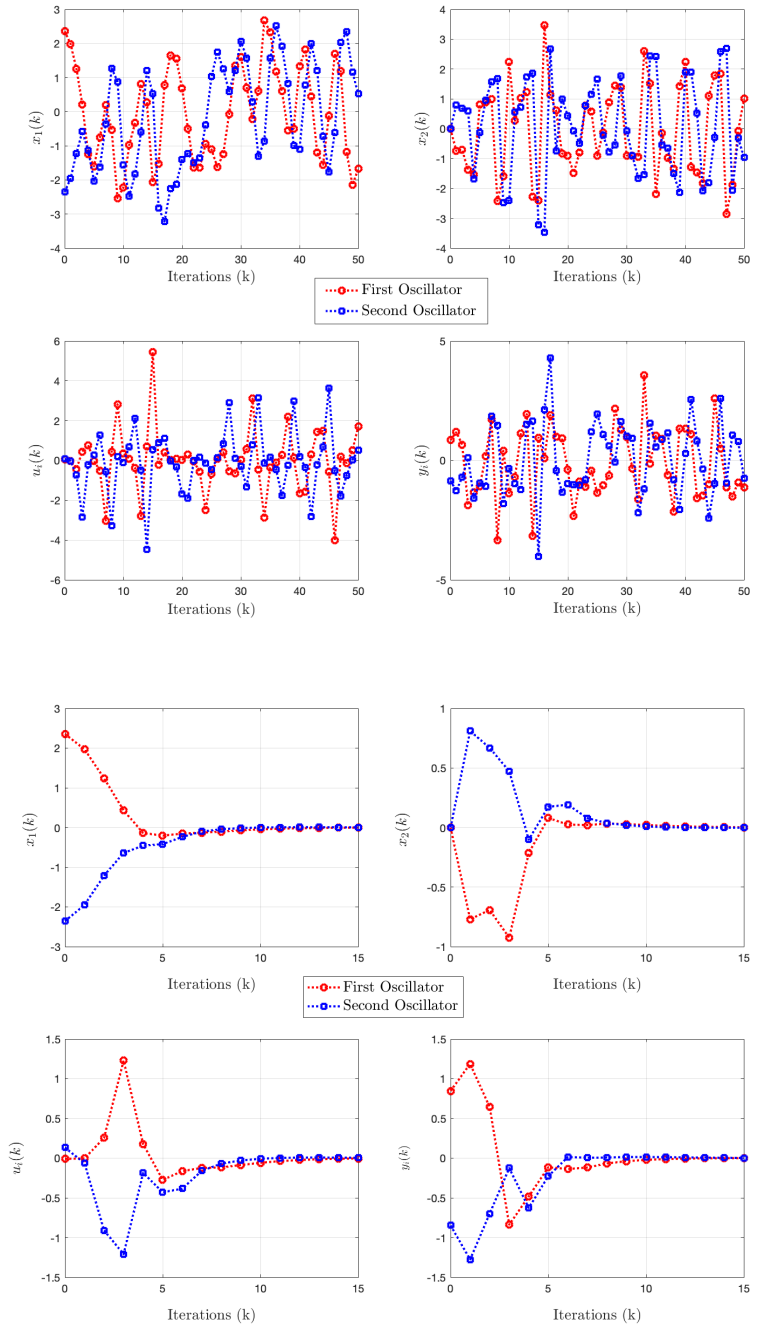

Fig. 2: Passive interconnection of two van der Pol oscillators: $v=0$ vs $=-\kappa h_{c, \bar{u}}^{a v}(x, \bar{u}(x)+v)$

$\mu_{2}=0.6$ and with initial conditions $x_{1}=\left(\frac{3}{4} \pi, 0\right), x_{2}=$ $\left(-\frac{3}{4} \pi, 0\right)$. More in details, for both the oscillators, we report the time evolutions of the states, the outputs $y_{i}=h_{i}\left(x_{i}\right)$ and the control actions. Two cases are reported for the interconnected system: $(i)$ only the power preserving interconnection feedback is applied (i.e., $v=0$ and $u=\bar{u}(x)$ as in (15)(16)); (ii) the power preserving interconnection feedback is complemented with a stabilizing damping feedback from the overall average output with $\kappa=1$. These simple simulations also let one understand that such a connection could bring to synchronization.

\section{CONCLUSIONS}

This paper formalizes the average dissipativity properties arising when interconnecting $u$-average passive discrete-time systems under suitable patterns preserving power exchanges. The methodologies apply to sampled-data systems as well. This study is preliminary to the development of new PBC strategies in discrete-time in terms of $\mathrm{CbI}$ and IDA-PBC by exploiting the $u$-average passivity properties of the interconnection. Further understanding of those properties in case of discrete port-Hamiltonian systems [22] is undergoing too.

\section{REFERENCES}

[1] J. C. Willems, "Dissipative dynamical systems part i: General theory," Archive for rational mechanics and analysis, vol. 45 , no. 5, pp. 321351, 1972.

[2] A. J. van der Schaft, L2-gain and passivity techniques in nonlinear control. Springer, 2000, vol. 2

[3] D. Hill and P. Moylan, "The stability of nonlinear dissipative systems," IEEE Transactions on Automatic Control, vol. 21, no. 5, pp. 708-711, 1976.

[4] J. C. Willems, "The behavioral approach to open and interconnected systems," IEEE Control Systems Magazine, vol. 27, no. 6, pp. 46-99, 2007.

[5] R. Ortega, A. J. Van Der Schaft, I. Mareels, and B. Maschke, "Putting energy back in control," IEEE Control Systems Magazine, vol. 21, no. 2, pp. 18-33, 2001

[6] R. Ortega, A. Van Der Schaft, F. Castanos, and A. Astolfi, "Control by interconnection and standard passivity-based control of porthamiltonian systems," IEEE Transactions on Automatic Control, vol. 53, no. 11, pp. 2527-2542, 2008.

[7] R. Ortega, A. Van Der Schaft, B. Maschke, and G. Escobar, "Interconnection and damping assignment passivity-based control of portcontrolled hamiltonian systems," Automatica, vol. 38, no. 4, pp. 585596, 2002.

[8] P. R. Giordano, A. Franchi, C. Secchi, and H. H. Bülthoff, "A passivity-based decentralized strategy for generalized connectivity maintenance," The International Journal of Robotics Research, vol. 32, no. 3, pp. 299-323, 2013.

[9] R. Reyes-Báez, A. van der Schaft, and B. Jayawardhana, "Passivity based distributed tracking control of networked euler-lagrange systems," IFAC-PapersOnLine, vol. 51, no. 23, pp. 136-141, 2018.

[10] J. Yao, Z.-H. Guan, and D. J. Hill, "Passivity-based control and synchronization of general complex dynamical networks," Automatica, vol. 45, no. 9, pp. 2107-2113, 2009.

[11] T. Hatanaka, N. Chopra, M. Fujita, and M. W. Spong, Passivity-based control and estimation in networked robotics. Springer, 2015.

[12] S. Monaco and D. Normand-Cyrot, "Nonlinear average passivity and stabilizing controllers in discrete time," Systems \& Control Letters, vol. 60 , no. 6 , pp. 431-439, 2011.

[13] _ , "Nonlinear representations and passivity conditions in discrete time," in Robustness in identification and control. Springer, 1999, pp. $422-433$.

[14] E. M. Navarro-López, "Several dissipativity and passivity implications in the linear discrete-time setting," Mathematical Problems in Engineering, vol. 2005, no. 6, pp. 599-616, 2005.

[15] M. Mattioni, S. Monaco, and D. Normand-Cyrot, "Sampled-data stabilization of feedforward dynamics with lyapunov cross-term," in 2016 IEEE 55th Conference on Decision and Control (CDC). IEEE, 2016, pp. 1322-1327.

[16] S. Monaco and D. Normand-Cyrot, "Discrete-time state representations, a new paradigm," in Perspectives in Control. Springer, 1998, pp. 191-203.

[17] C. I. Byrnes and W. Lin, "Discrete-time lossless systems, feedback equivalence and passivity," in Proceedings of 32nd IEEE Conference on Decision and Control. IEEE, 1993, pp. 1775-1781.

[18] M. Mattioni, S. Monaco, and D. Normand-Cyrot, "Feedforwarding under sampling," IEEE Transactions on Automatic Control, vol. 64, no. 11, 2019.

[19] — - "Forwarding stabilization in discrete time," Automatica, vol. 109, p. 108532, 2019. [Online]. Available: http://www.sciencedirect.com/science/article/pii/S0005109819303930

[20] V. Talasila, J. Clemente-Gallardo, and A. Van der Schaft, "Discrete port-hamiltonian systems," Systems \& Control Letters, vol. 55, no. 6, pp. 478-486, 2006.

[21] Y. Yalçin, L. G. Sümer, and S. Kurtulan, "Discrete-time modeling of hamiltonian systems," Turkish Journal of Electrical Engineering \& Computer Sciences, vol. 23, no. 1, pp. 149-170, 2015.

[22] A. Moreschini, M. Mattioni, S. Monaco, and D. Normand-Cyrot, "Discrete port-controlled hamiltonian dynamics and average passivation," in 58th IEEE Conference on Decision and Control (CDC), December 2019. 